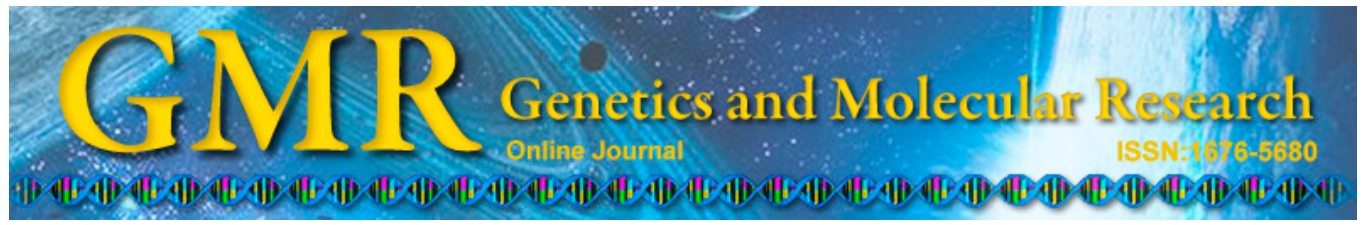

\title{
Bioinformatic analysis of miRNA expression patterns in TFF2 knock-out mice
}

\author{
Y. Yin, H.Q. Shan, W. Huang, Y.M. Wu, H. Lu and Y. Jin \\ Department of Spinal Surgery, \\ Jiangsu College Affiliated to the Kunshan First People's Hospital, \\ Kunshan, Jiangsu Province, China \\ Corresponding author: Y. Yin \\ E-mail: yy_19723@hotmail.com
}

Genet. Mol. Res. 13 (4): 8502-8510 (2014)

Received October 7, 2013

Accepted June 6, 2014

Published October 20, 2014

DOI http://dx.doi.org/10.4238/2014.October.20.26

\begin{abstract}
Trefoil factors, which bear a unique 3-loop trefoil domain, are a family of small secretory protease-resistant peptides (7-12 kDa) discovered in the $1980 \mathrm{~s}$. Trefoil factor 2 (TFF2) is a unique member of trefoil factors family that plays important roles in gastrointestinal mucosal defense and repair. However, few studies have characterized the miRNA expression patterns in TFF2 knock-out mice. In this study, we investigated the regulatory role of miRNAs in TFF2 knock-out mice. Whole miRNome profiling for TFF2 knock-out mice and wild-type mice were downloaded from the Gene Expression Omnibus database. A total of 14 differentially expressed miRNAs were identified using the limma package. Target genes for 2 differentially expressed miRNAs were retrieved from 2 databases. After mapping these target genes into STRING, an interaction network was constructed. Gene Ontology analysis suggested that the differentially expressed miRNAs are involved in cyclic AMP metabolism and the growth process. Additionally, dysregulated miRNAs target pathways of transforming growth factor-beta signaling pathway and cytokinecytokine receptor interaction. Our results suggest that miRNAs may play important regulatory roles in processes involving TFF2,
\end{abstract}


particularly in the regulation of signal transduction pathways. However, further validation of our results is needed.

Key words: Interaction network; MicroRNA; Trefoil factor 2 knock-out

\section{INTRODUCTION}

Trefoil factors (TFFs), which bear a unique 3-loop trefoil domain, are a family of small secretory protease-resistant peptides (7-12 kDa in mammals) discovered by Thim (1989) and Thim et al. (1982). TFFs play crucial roles in gastrointestinal mucosal defense and repair (Wong et al., 1999; Taupin and Podolsky, 2003; Kjellev, 2009). These peptides are thought to serve as growth factors and were found to be overexpressed during inflammatory processes and the progression of some tumors, including in the esophagus, breast, colon, prostate, and pancreas (Emami et al., 2004). Ectopic expression of TFFs in various carcinomas may be a useful prognostic marker (Yamachika et al., 2002; Kosriwong et al., 2011). To date, 3 members of the TFF family have been annotated in mammals, including TFF1, TFF2, and TFF3. Among these, TFF2 may be involved in the restoration of injured mucosa by increasing cellular motility and its rapid induction in the vicinity of injured mucosa (Alison et al., 1995).

TFF2 is the first member identified of the TFF family and contains 2 trefoil motifs (Thim et al., 1982). It is expressed and secreted preferentially by gastric mucous neck cells (Lefebvre et al., 1993; Jeffrey, 1994) and is upregulated under diverse pathologic conditions in the gastrointestinal tract, such as chronic inflammation and gut injury (Wright et al., 1993; Hoffmann and Jagla, 2002). Previous studies identified several roles for TFF2. For example, TFF2 may inhibit gastric acid secretion (Thim et al., 1982; Konturek et al., 1997) and several colon cancer cell lines (Hoosein et al., 1989). Additionally, roles in restitution and stabilization of the mucin gel layer have been proposed (Tanaka et al., 1997). MicroRNAs (miRNAs) are short, non-coding RNAs ( 22 nucleotides) that are typically involved in posttranscriptional regression by binding to partially complementary sites in the 3 -untranslated region of their mRNA targets (Lee et al., 1993; Wightman et al., 1993; Hua et al., 2009). Increasing evidence suggests that target mRNAs can be degraded by miRNAs, which are also important in the processes of tumorigenesis and cancer development (Lim et al., 2005; Wang and Wang, 2006). However, few studies have characterized the miRNA expression patterns in TFF2 knock-out mice.

Although controlling gene activity by miRNAs has received increasing attention, thousands of regulatory transcripts still require analysis, particularly selection of targets, mechanisms of complementarity, and cooperativity of miRNAs. Predicting miRNA targets is a first-line approach to understanding these complex systems and can facilitate experimental validation of such mechanisms of gene control (Shah and Blin, 2010). In this study, we analyzed the miRNA expression profile of TFF2 knock-out mice and wild-type mice downloaded from the Gene Expression Omnibus database. MiRecords and miRTarBase were used to obtain genes correlated with miRNAs. WebGestalt was used to analyze the function enrichment and to identify the over-represented gene ontology (GO) categories and pathways of genes in the interaction network at a false discovery rate (FDR) of 0.05 , and identify enriched pathways. These results will enable exploration of the functions of miRNAs involved in regulating signal transduction pathways in TFF2 knock-out mice. 


\section{MATERIAL AND METHODS}

\section{Microarray data}

The miRNA expression profile data on TFF2 knock-out mice with wild type controls were downloaded from Gene Expression Omnibus database, which were deposited by Shah et al. (2012) (accession No. GSE25815). The febit Mus musculus miRBase V14 platform (GPL10849) was used to analyze miRNA expression profiles. A total of 12 gene chips were available, including 6 gene chips from wild-type mice and 6 from TFF2 knock-out mice. We downloaded the raw CEL data and the annotation information for the platform.

\section{Data preprocessing and identification of differentially expressed miRNAs}

The Affy package in R (Gautier et al., 2004; R Development Core Team, 2012) was used to convert the CEL data into expression estimates. Missing measurements were imputed (Troyanskaya et al., 2001). Next, background correction and normalization were conducted by the robust multi-array average algorithm (Irizarry et al., 2003). The linear models for microarray data (limma) package (Smyth, 2004) in R was used to identify differentially expressed miRNAs between wild-type mice and TFF2 knock-out mice. In a recent study, Jeanmougin et al. (2010) compared 8 tests representative of variance modeling strategies and demonstrated that limma presents several practical advantages compared with other methods. The Multtest package was used to perform multiple test correction. FDR was the expected proportion of false positive findings among the declared significant results, which was liberal and more powerful than FWER, a stricter algorithm in some situations. FDR $<0.05$ and $|\log \mathrm{FC}|>1$ were selected as cut-off criteria and has been used in several previous studies (Zhang et al., 2013; Walsh et al., 2014).

\section{Predicting target genes of differentially expressed miRNAs}

Two miRNA databases, miRecords and miRTarBase, were used to predict target genes of differentially expressed miRNAs. The miRecords database hosts 2286 records of experimentally validated miRNA-target interactions between 548 miRNAs and 1579 target genes in 9 animal species (Xiao et al., 2009). The miRTarBase database (Release 3.5) curates 4876 experimentally verified miRNA-target interactions between 726 miRNAs and 2789 target genes among 17 species (Hsu et al., 2011). We predicted target genes of differentially expressed miRNAs in the 2 databases and extracted the data for the predicted target genes.

\section{Construction of interaction network}

The Search Tool for the Retrieval of Interacting Genes (STRING) database provides both experimental validated and predicted interaction information (Szklarczyk et al., 2011). STRING 9.0 covers more than 1100 completely sequenced organisms. We 
searched the interactions among target genes of differentially expressed miRNAs using the online search tool in STRING and constructed an interaction network.

\section{Function enrichment analysis}

WebGestalt is a web-based integrated data mining system composed of 4 modules: gene set management, information retrieval, organization/visualization, and statistics. The organization/visualization module organizes and visualizes gene sets in various biological contexts, including GO, tissue expression pattern, and protein domain information (Zhang et al., 2005; Duncan et al., 2010). In this study, we used WebGestalt to identify over-represented GO categories and pathways of genes in the interaction network at an FDR of 0.05 .

\section{RESULTS}

\section{Identification of differentially expressed miRNAs}

The limma package in $\mathrm{R}$ was used to identify the differentially expressed miRNAs between wild-type mice and TFF2 knock-out mice. After multiple test correction, 14 differentially expressed miRNAs were identified at cutoff of FDR $<0.05$ and $|\operatorname{logFC}|>1$. Three miRNAs were upregulated, while the remaining 11 miRNAs were downregulated (Table 1).

\begin{tabular}{lcc}
\multicolumn{2}{c}{ Table 1. Differentially expressed miRNAs $(*$ FDR $<0.05)$} & \\
\hline miRNA & FDR & LogFC \\
\hline mmu-miR-26b* & 0.009765 & 2.577116 \\
mmu-miR-142-3p & 0.015303 & 2.510031 \\
mmu-miR-125a-5p & 0.008138 & 1.313717 \\
mmu-miR-490 & 0.009795 & -1.02558 \\
mmu-miR-1899 & 0.001542 & -1.07157 \\
mmu-miR-712* & 0.000507 & -1.11783 \\
mmu-miR-715 & 0.001851 & -1.16979 \\
mmu-miR-488* & 0.004491 & -1.23071 \\
mmu-miR-1190 & 0.000494 & -1.30295 \\
mmu-miR-1895 & 0.001279 & -1.50334 \\
mmu-miR-29b* & 0.006877 & -2.00355 \\
mmu-miR-876-5p & 0.013368 & -2.24011 \\
mmu-miR-692 & 0.040429 & -2.61369 \\
mmu-miR-463* & 0.031052 & -2.66591 \\
\hline
\end{tabular}

\section{Predicting target genes of differentially expressed miRNAs}

We retrieved the target genes of the differentially expressed miRNAs from the databases of miRecords and miRTarBase (Table 2). After predicting putative miRNA target genes in each database, we extracted target genes shared by the 2 databases. As shown in Table 2 , we obtained the target genes for mmu-miR-142-3p and mmu-miR-29b. The target gene for mmu-miR-142-3p was ADCY9 and the target genes of mmu-miR-29b were COL5A3, DUSP2, COL1A1, COL4A2, HDAC4, TGFB3, and ACVR2A. 
Table 2. Target genes of differentially expressed miRNAs.

\begin{tabular}{lll}
\hline miRNA & miRecords & miRTarBase \\
\hline mmu-miR-26b* & - & LEF1 \\
mmu-miR-142-3p & ADCY9 & ADCY9 \\
mmu-miR-125a-5p & - & CBX7, TRIM71 \\
mmu-miR-490 & - & - \\
mmu-miR-1899 & - & - \\
mmu-miR-712* & - & - \\
mmu-miR-715 & - & - \\
mmu-miR-488* & - & - \\
mmu-miR-1190 & - & - \\
mmu-miR-1895 & COL5A3, DUSP2, COL1A1, COL4 & ACVR2A, BAK1, BBC3, BCL2L11, BMF, CO1A1, \\
mmu-miR-29b* & & COL1A2, COL3A1, COL4A2, COL5A3, CTNNB \\
& - & IP1, DNMT3A, DNMT3B, DUSP2, ELN, FBN1, \\
mmu-miR-876-5p & - & - \\
mmu-miR-692 & - & - \\
mmu-miR-463* & & - \\
\hline
\end{tabular}

*The lower expressed miR produced. Gene names in bold mean these genes are presented in both miRecords and miRTarBase.

\section{Construction of interaction network}

We retrieved all interactions of target genes of differentially expressed miRNAs using the online search tool STRING and constructed an interaction network (Figure 1).

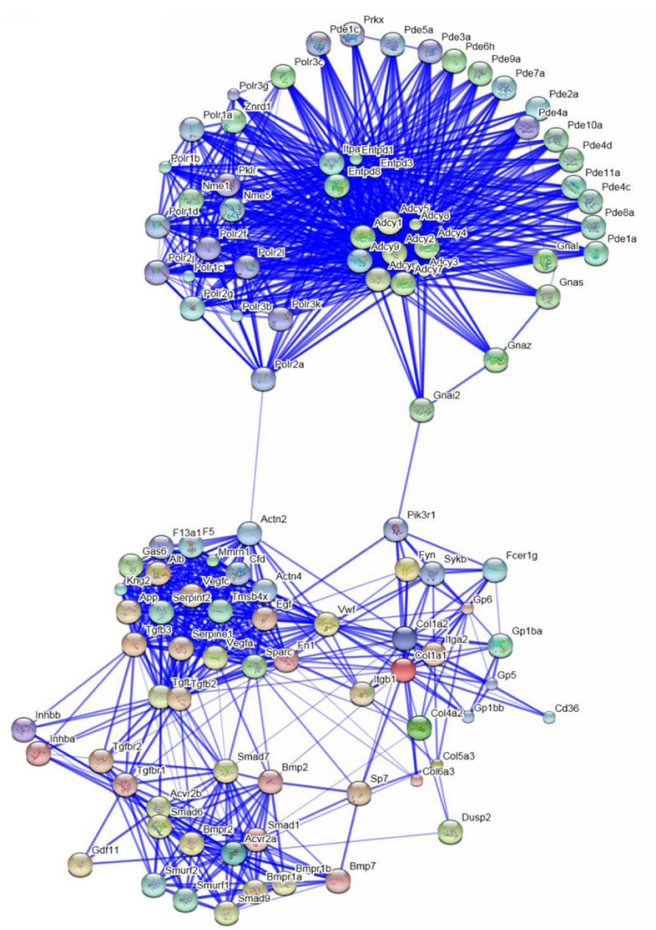

Figure 1. Interaction network constructed by target genes of differentially expressed miRNAs. Circles of different colors stand for different genes. Blue lines link two related genes. 


\section{Function enrichment analysis}

To investigate the functions of the genes in the interaction network, we performed GO and KEGG pathway enrichment analysis using WebGestalt. At an FDR of 0.05, 13 GO categories were enriched, including cAMP metabolic process, cAMP biosynthetic process, bone morphogenetic protein signaling pathway and skeletal system development (Table 3). Two pathways were significantly enriched, including the transforming growth factor-beta signaling pathway and cytokine-cytokine receptor interaction (Table 4).

\begin{tabular}{|c|c|c|c|}
\hline GO-ID & Term & Count & FDR \\
\hline GO:0046058 & cAMP metabolic process & 11 & $4.21 \mathrm{E}-15$ \\
\hline GO:0006171 & cAMP biosynthetic process & 9 & 7.33E-12 \\
\hline GO:0030817 & regulation of cAMP biosynthetic process & 11 & $2.98 \mathrm{E}-08$ \\
\hline GO:0030814 & regulation of cAMP metabolic process & 11 & 4.08E-08 \\
\hline GO:0030509 & BMP signaling pathway & 8 & $2.90 \mathrm{E}-07$ \\
\hline GO:0001501 & skeletal system development & 6 & $1.38 \mathrm{E}-06$ \\
\hline GO:0007179 & transforming growth factor beta receptor signaling pathway & 9 & $1.76 \mathrm{E}-06$ \\
\hline GO:0040007 & growth & 12 & $1.30 \mathrm{E}-04$ \\
\hline GO:0048705 & skeletal system morphogenesis & 8 & 0.04922 \\
\hline GO:0004114 & 3', 5'-cyclic-nucleotide phosphodiesterase activity & 14 & 4.21E-19 \\
\hline GO:0005024 & transforming growth factor beta receptor activity & 7 & $6.29 \mathrm{E}-07$ \\
\hline GO:0047555 & 3', 5'-cyclic-GMP phosphodiesterase activity & 6 & $1.34 \mathrm{E}-05$ \\
\hline GO:0008083 & growth factor activity & 11 & $1.52 \mathrm{E}-04$ \\
\hline
\end{tabular}

Table 4. Enriched KEGG pathways $(\mathrm{FDR}<0.05)$.
\begin{tabular}{llcc} 
& & \\
\hline Pathway ID & Term & Count & FDR \\
\hline mmu 04350 & TGF-beta signaling pathway & 20 & $1.22 \mathrm{E}-13$ \\
mmu04060 & Cytokine-cytokine receptor interaction & 17 & 0.00358 \\
\hline
\end{tabular}

\section{DISCUSSION}

miRNAs are post-transcriptional regulators that bind to complementary sequences on target mRNAs, typically resulting in translational repression or target degradation and gene silencing (Kusenda et al., 2006; Bartel, 2009). In this study, we investigated the miRNA expression profiles of TFF2 knock-out mice and wild-type mice. A total of 14 differentially expressed miRNAs were found to be involved in regulating TFF2 gene activity.

The original study of this dataset by Shah et al. (2012) identified a total of 48 differentially expressed miRNAs, including 26 downregulated and 22 upregulated miRNAs. The number of differentially expressed miRNAs in our study was lower than in the previous study. Among the 14 differentially expressed miRNAs, 7 miRNAs were identified in the original paper, while the other 7 miRNAs were newly identified as being differently expressed (mmu-miR-26b*, mmu-miR-142-3p, mmu-miR-1190, mmu-miR-29b*, mmu-miR-463*, mmu-miR-692, and mmu-miR-876-5p). There are 2 explanations for this result. First, the cutoff criteria for identifying differentially expressed miRNAs were different. In our study, we chose an FDR cutoff of $<0.05$ and $|\operatorname{logFC}|>1$, which was stricter than in the original study, to achieve more reliable results. Second, different statistical methods for identifying differentially expressed genes leads to different results (Abruzzo et al., 2005). In this study, we used 
the limma package, which has advantage in studies with small sample sizes compared to other methods (Jeanmougin et al., 2010), for analyzing the miRNA expression profile.

Because miRNAs play an important role in posttranscriptional regression by targeting mRNAs, we examined the function of differentially expressed miRNAs by identifying putative targets. However, most target genes of miRNA were computational predictions with only limited overlap and lacking experimental confirmations (Jovanovic et al., 2010). To reduce the identification of false-positives, we only extracted the target genes existing in the 2 databases. One target gene of mmu-miR-142-3p and 7 target genes of mmu-miR-29b were retrieved. An interaction network was constructed by mapping these target genes into the STRING database.

Function enrichment analysis showed that most of the dysregulated GO categories were associated with cAMP. cAMP is a second messenger that functions in intracellular signal transduction in many different organisms. Previous studies have suggested that dysregulation of cAMP pathways and aberrant activation of cAMP-controlled genes are linked to the progression of some cancers (Simpson et al., 1996; Abramovitch et al., 2004; Dumaz et al., 2006). Studies have also suggested that TFF2 may be involved in cancer progression (Jung et al., 2010). Thus, TFF2 may be involved in cancer progression by regulating cAMP metabolism.

\section{CONCLUSIONS}

In conclusion, we identified 14 significant dysregulated miRNAs in TFF2 knock-out mice. These miRNAs may be involved in regulating signaling transduction pathways and growth processes. Although there were some limitations to our study, our results are important for understanding the roles of miRNAs in TFF2 knock-out mice.

Because our results were based on microarray data derived from a small sample size, further validation is required. Although the selective conditions were stricter than those used in the original paper, quantitative polymerase chain reaction results were not available. An integrative approach comparing miRNA data with mRNA data should be used to supplement the relationship between miRNA and mRNA.

\section{REFERENCES}

Abramovitch R, Tavor E, Jacob-Hirsch J, Zeira E, et al. (2004). A pivotal role of cyclic AMP-responsive element binding protein in tumor progression. Cancer Res. 64: 1338-1346.

Abruzzo LV, Wang J, Kapoor M, Medeiros LJ, et al. (2005). Biological validation of differentially expressed genes in chronic lymphocytic leukemia identified by applying multiple statistical methods to oligonucleotide microarrays. $J$. Mol. Diagn. 7: 337-345.

Alison MR, Chinery R, Poulsom R, Ashwood P, et al. (1995). Experimental ulceration leads to sequential expression of spasmolytic polypeptide, intestinal trefoil factor, epidermal growth factor and transforming growth factor alpha mRNAs in rat stomach. J. Pathol. 175: 405-414.

Bartel DP (2009). MicroRNAs: target recognition and regulatory functions. Cell 136: 215-233.

Dumaz N, Hayward R, Martin J, Ogilvie L, et al. (2006). In melanoma, RAS mutations are accompanied by switching signaling from BRAF to CRAF and disrupted cyclic AMP signaling. Cancer Res. 66: 9483-9491.

Duncan D, Prodduturi N and Zhang B (2010). WebGestalt2: an updated and expanded version of the Web-based Gene Set Analysis Toolkit. BMC Bioinformatics 11 (Suppl 4): 10.

Emami S, Rodrigues S, Rodrigue CM, Le Floch N, et al. (2004). Trefoil factor family (TFF) peptides and cancer progression. Peptides 25: 885-898.

Gautier L, Cope L, Bolstad BM and Irizarry RA (2004). affy-analysis of Affymetrix GeneChip data at the probe level. Bioinformatics 20: 307-315.

Hoffmann W and Jagla W (2002). Cell type specific expression of secretory TFF peptides: colocalization with mucins and 
synthesis in the brain. Int. Rev. Cytol. 213: 147-181.

Hoosein NM, Thim L, Jorgensen KH and Brattain MG (1989). Growth stimulatory effect of pancreatic spasmolytic polypeptide on cultured colon and breast tumor cells. FEBS Lett. 247: 303-306.

Hsu SD, Lin FM, Wu WY, Liang C, et al. (2011). miRTarBase: a database curates experimentally validated microRNAtarget interactions. Nucleic Acids Res. 39: D163-D169.

Hua YJ, Tang ZY, Tu K, Zhu L, et al. (2009). Identification and target prediction of miRNAs specifically expressed in rat neural tissue. BMC Genomics 10: 214.

Irizarry RA, Hobbs B, Collin F, Beazer-Barclay YD, et al. (2003). Exploration, normalization, and summaries of high density oligonucleotide array probe level data. Biostatistics 4: 249-264.

Jeanmougin M, de Reynies A, Marisa L, Paccard C, et al. (2010). Should we abandon the t-test in the analysis of gene expression microarray data: a comparison of variance modeling strategies. PLoS One 5: e12336.

Jeffrey GP, Oates PS, Wang TC, Babyatsky MW, et al. (1994). Spasmolytic polypeptide: a trefoil peptide secreted by rat gastric mucous cells Gastroenterology 106: 336-345.

Jovanovic M, Reiter L, Picotti P, Lange V, et al. (2010). A quantitative targeted proteomics approach to validate predicted microRNA targets in C. elegans. Nat. Methods 7: 837-842.

Jung K, Kim P, Kim J, Noh J, et al. (2010). Decreased expression of TFF2 and gastric carcinogenesis. Mol. Cell. Toxicol. 6: 261-269.

Kjellev S (2009). The trefoil factor family - small peptides with multiple functionalities. Cell Mol. Life Sci. 66: 1350-1369.

Konturek PC, Brzozowski T, Konturek SJ, Elia G, et al. (1997). Role of spasmolytic polypeptide in healing of stressinduced gastric lesions in rats. Regul. Pept. 68: 71-79.

Kosriwong K, Menheniott TR, Giraud AS, Jearanaikoon P, et al. (2011). Trefoil factors: tumor progression markers and mitogens via EGFR/MAPK activation in cholangiocarcinoma. World J. Gastroenterol. 17: 1631-1641.

Kusenda B, Mraz M, Mayer J and Pospisilova S (2006). MicroRNA biogenesis, functionality and cancer relevance. Biomed. Pap. Med. Fac. Univ. Palacky Olomouc Czech Repub. 150: 205-215.

Lee RC, Feinbaum RL and Ambros V (1993). The C. elegans heterochronic gene lin-4 encodes small RNAs with antisense complementarity to lin-14. Cell 75: 843-854.

Lefebvre O, Wolf C, Kedinger M, Chenard MP, et al. (1993). The mouse one P-domain (pS2) and two P-domain (mSP) genes exhibit distinct patterns of expression. J. Cell Biol. 122: 191-198.

Lim LP, Lau NC, Garrett-Engele P, Grimson A, et al. (2005). Microarray analysis shows that some microRNAs downregulate large numbers of target mRNAs. Nature 433: 769-773.

R Development Core Team (2012). R: A Language and Environment for Statistical Computing. R Foundation for Statistical Computing, Vienna.

Shah A and Blin N (2010). Target sequences for microRNAs within the Tff gene cluster. BioChip J. 4: 279-284.

Shah AA, Leidinger P, Keller A, Wendschlag A, et al. (2012). Altered miRNA expression patterns in Tff2 knock-out mice correlate with cellular pathways of neoplastic development and caloric metabolism. Int. J. Mol. Med. 29: 637-643.

Simpson BJ, Ramage AD, Hulme MJ, Burns DJ, et al. (1996). Cyclic adenosine 3',5'-monophosphate-binding proteins in human ovarian cancer: correlations with clinicopathological features. Clin. Cancer Res. 2: 201-206.

Smyth GK (2004). Linear models and empirical bayes methods for assessing differential expression in microarray experiments. Stat. Appl. Genet. Mol. Biol. 3: Article3.

Szklarczyk D, Franceschini A, Kuhn M, Simonovic M, et al. (2011). The STRING database in 2011: functional interaction networks of proteins, globally integrated and scored. Nucleic Acids Res. 39: D561-D568.

Tanaka S, Podolsky DK, Engel E, Guth PH, et al. (1997). Human spasmolytic polypeptide decreases proton permeation through gastric mucus in vivo and in vitro. Am. J. Physiol. 272: G1473-G1480.

Taupin D and Podolsky DK (2003). Trefoil factors: initiators of mucosal healing. Nat. Rev. Mol. Cell Biol. 4: 721-732.

Thim L, Jorgensen KH and Jorgensen KD (1982). Pancreatic spasmolytic polypeptide (PSP): II. Radioimmunological determination of PSP in porcine tissues, plasma and pancreatic juice. Regul. Pept. 3: 221-230.

Thim L (1989). A new family of growth factor-like peptides. 'Trefoil' disulphide loop structures as a common feature in breast cancer associated peptide (pS2), pancreatic spasmolytic polypeptide (PSP), and frog skin peptides (spasmolysins). FEBS Lett. 250: 85-90.

Troyanskaya O, Cantor M, Sherlock G, Brown P, et al. (2001). Missing value estimation methods for DNA microarrays. Bioinformatics 17: 520-525.

Walsh J, Jenkins RE, Wong M, Olayanju A, et al. (2014). Identification and quantification of the basal and inducible Nrf2dependent proteomes in mouse liver: biochemical, pharmacological and toxicological implications. J. Proteomics 108: 171-187.

Wang X and Wang X (2006). Systematic identification of microRNA functions by combining target prediction and expression profiling. Nucleic Acids Res. 34: 1646-1652. 
Wightman B, Ha I and Ruvkun G (1993). Posttranscriptional regulation of the heterochronic gene lin-14 by lin-4 mediates temporal pattern formation in C. elegans. Cell 75: 855-862.

Wong WM, Poulsom R and Wright NA (1999). Trefoil peptides. Gut 44: 890-895.

Wright NA, Poulsom R, Stamp G, Van Noorden S, et al. (1993). Trefoil peptide gene expression in gastrointestinal epithelial cells in inflammatory bowel disease. Gastroenterology 104: 12-20.

Xiao F, Zuo Z, Cai G, Kang S, et al. (2009). miRecords: an integrated resource for microRNA-target interactions. Nucleic Acids Res. 37: D105-D110.

Yamachika T, Werther JL, Bodian C, Babyatsky M, et al. (2002). Intestinal trefoil factor: a marker of poor prognosis in gastric carcinoma. Clin. Cancer Res. 8: 1092-1099.

Zhang B, Kirov S and Snoddy J (2005). WebGestalt: an integrated system for exploring gene sets in various biological contexts. Nucleic Acids Res. 33: W741-W748.

Zhang YD, Gu XH, Dai RY, Chen Z, et al. (2013). Screening of differentially expressed genes of middle cerebral artery occlusion with DNA microarray. Eur. Rev. Med. Pharmacol. Sci. 17: 1205-1210. 